\title{
Oxidized LDL reduces monocyte CCR2 expression through pathways involving peroxisome proliferator-activated receptor $\gamma$
}

\author{
Ki Hoon Han, Mi Kyung Chang, Agnes Boullier, Simone R. Green, Andrew Li, \\ Christopher K. Glass, and Oswald Quehenberger
}

Department of Medicine, University of California at San Diego, La Jolla, California, USA

Address correspondence to: Oswald Quehenberger, Department of Medicine, 0682, University of California at San Diego, 9500 Gilman Drive, La Jolla, California 92093-0682, USA.

Phone: (858) 534-4401; Fax: (858) 534-2005; E-mail: oquehenberger@ucsd.edu.

Received for publication April 7, 2000, and accepted in revised form July 18, 2000.

The CCR2-mediated recruitment of monocytes into the vessel wall plays an important role in all stages of atherosclerosis. In recent studies, we have shown that lipoproteins can modulate CCR2 expression and have identified native LDL as a positive regulator. In contrast, oxidized LDL (OxLDL), which is mainly formed in the aortic intima, reduces CCR2 expression, promotes monocyte retention, and may cause pathological accumulation of monocytes in the vessel wall. We now provide evidence that OxLDL reduces monocyte CCR2 expression by activating intracellular signaling pathways that may involve peroxisome proliferator-activated receptor $\gamma$ (PPAR $\gamma$ ). Receptormediated uptake of the lipoprotein particle was required and allows for delivery of the exogenous ligand to the nuclear receptor. The suppression of CCR2 expression by OxLDL was mediated by lipid components of OxLDL, such as the oxidized linoleic acid metabolites 9-HODE and 13-HODE, known activators of PPAR $\gamma$. Modified apoB had no such effect. Consistent with a participation of the PPAR $\gamma$ signaling pathway, BRL49653 reduced CCR2 expression in freshly isolated human monocytes ex vivo and in circulating mouse monocytes in vivo. These results implicate PPAR $\gamma$ in the inhibition of CCR2 gene expression by oxidized lipids, which may help retain monocytes at sites of inflammation, such as the atherosclerotic lesion.

J. Clin. Invest. 106:793-802 (2000).

\section{Introduction}

The accumulation of lipid-laden foam cells is characteristic for developing atherosclerotic lesions. Most of these cells are recruited from the circulation and take up residence in the vessel wall where they ingest massive amounts of lipids to become foam cells. Blood monocytes are the primary source of arterial macrophages, and there is strong evidence that the monocyte chemoattractant protein-1 (MCP-1) is involved in the recruitment process $(1,2)$. MCP- 1 is expressed and secreted by the cells of the vessel wall in response to a variety of proinflammatory stimuli, and the presence of MCP- 1 in atherosclerotic lesions is well documented (3-9). More direct evidence for a role of MCP-1 in atherosclerosis came from mouse models demonstrating that MCP-1 deficiency reduced atherosclerosis, whereas overexpression of MCP-1 accelerated atherosclerosis (10-12).

MCP-1 is a member of the CC family of chemokines and was isolated on the basis of its potent chemoattractant activity for monocytes $(13,14)$. The effect of MCP- 1 is mediated by the seven-transmembrane-spanning $G$ protein-coupled receptor CCR2, which is expressed mainly in monocytes, basophils, and certain subsets of T cells $(15,16)$. The functional interaction between CCR2 and MCP- 1 is complex and is consistent with a multidomain binding model $(17,18)$. The nonredundant function of CCR2 in host defense and its important role in atherogenesis was demonstrated recently in animal models (19-21).

If a reduction in CCR2 expression decreases lesion formation, then any increase in expression should accelerate atherosclerosis. In a recent study we demonstrated that CCR2 expression is significantly increased in circulating monocytes in hypercholesterolemic humans (22). The level of CCR2 expression correlated directly with the plasma levels of LDL and inversely with the plasma concentrations of HDL. The increase in CCR2 expression enhanced the chemotactic response to MCP- 1 and may promote the initial recruitment of monocytes to atherosclerotic lesions. In contrast to native LDL, oxidized LDL (OxLDL) caused a rapid downregulation of CCR2 expression, and these monocytes no longer responded to MCP-1. Plasma LDL can cross the endothelium and accumulate in the intima where it may undergo oxidative modification to acquire a number of biological properties that are potentially proatherogenic $(23,24)$. As a negative regulator of monocyte CCR2 expression, it may promote the arrest of newly recruited monocytes in the arterial wall, allowing for 
their cytokine- and growth factor-induced maturation to macrophages (25).

Peroxisome proliferator-activated receptor $\gamma(\operatorname{PPAR} \gamma)$ is a member of the nuclear receptor superfamily that functions as ligand-dependent transcription factor. PPAR $\gamma$ is abundantly expressed in adipose tissue and appears to play an important role in adipose differentiation and lipid metabolism $(26,27)$. It may also be the target for some thiazolidinediones that are used for the treatment of type 2 diabetes $(28,29)$. In addition to adipocytes, PPAR $\gamma$ is also expressed in monocytes/macrophages and has been implicated in the regulation of macrophage inflammatory responses (30-32).

The present study was designed to investigate the mechanisms responsible for the reduction of monocyte CCR2 expression by OxLDL. Internalization of the lipoprotein particle was required, and the inhibition of CCR2-gene expression was mediated by the lipids from OxLDL but not by the isolated apoB. OxLDL is rich in oxidized polyunsaturated fatty acids, which are known ligands for PPAR $\gamma$, and there is strong evidence that the OxLDL-mediated inhibition of monocyte CCR2 expression involves PPAR $\gamma$ dependent intracellular signaling events.

\section{Methods}

Reagents and Ab's. Linoleic acid and TNF- $\alpha$ were obtained from Sigma Chemical Co. (St. Louis, Missouri, USA). 1-Palmitoyl 2-arachidonoyl phosphatidylcholine (PAPC) was from Avanti Polar Lipids (Alabaster, Alabama, USA), and 9-hydroxyoctadecadienoic acid (9-HODE) and 13-HODE were from Cayman Chemical Co. (Ann Arbor, Michigan, USA). Dibutyl phthalate and dioctyl phthalate were purchased from Aldrich Chemical Co. (Milwaukee, Wisconsin, USA), and 3,3'-dihexadecyloxacarbocyanine perchlorate (DiO) was from Molecular Probes (Eugene, Oregon, USA). BRL49653 was from Glaxo Wellcome (Research Triangle Park, North Carolina, USA). Recombinant MCP-1 was purchased from R\&D Systems Inc. (Minneapolis, Minnesota, USA), and ${ }^{125} \mathrm{I}-$ MCP-1 (specific activity, $2200 \mathrm{Ci} / \mathrm{mmol}$ ) was from DuPont NEN (Boston, Massachusetts, USA). The neutralizing mouse anti-human CD36 IgM, OKM5, was purchased from Sigma Chemical Co. Phycoerythrinconjugated mouse anti-human CCR2 IgG and nonspecific IgG were obtained from R\&D Systems Inc., and unlabeled mouse and human nonspecific IgG was from Sigma Chemical Co. Fluorescein isothiocyanate-conjugated mouse anti-human CD14 IgG and rat anti-mouse CD80 IgG were from PharMingen (San Diego, California, USA). Taq polymerase and deoxynucleotide triphosphates were from Promega (Madison, Wisconsin, USA).

Animals, cell culture, and preparation of monocytes. THP-1 cells (American Type Culture Collection, Manassas, Virginia, USA) were maintained in RPMI-1640 medium (BioWhittaker, Walkersville, Maryland, USA), supplemented with 10\% low-endotoxin FCS (Hyclone, Logan,
Utah, USA), $100 \mathrm{U} / \mathrm{mL}$ penicillin, and $100 \mu \mathrm{g} / \mathrm{mL}$ streptomycin (Irvine Scientific, Santa Ana, California, USA). To avoid activation and differentiation, the concentration of THP- 1 cells never exceeded $0.5 \times 10^{6}$ cells $/ \mathrm{mL}$.

Human monocytes were isolated as described earlier (22). Briefly, $100 \mathrm{~mL}$ of whole blood was drawn into tubes containing $3 \mathrm{mM}$ EDTA, the buffy coat was isolated by centrifugation at $1,500 \mathrm{~g}$ for 30 minutes at $4^{\circ} \mathrm{C}$, carefully layered onto an equal volume of Histopaque 1077 (Sigma Chemical Co.), and mononuclear cells were isolated by centrifugation at $400 \mathrm{~g}$ for 30 minutes at room temperature. The cell pellet was washed twice with PBS containing $0.02 \%$ EDTA, and the cells were plated into a $150-\mathrm{mm}$ dish containing RPMI-1640 medium supplemented with $10 \%$ autologous serum. After 1 hour of incubation at $37^{\circ} \mathrm{C}$, nonadhering cells were removed by washing the plate twice with PBS, and the adhering monocytes were harvested by brief trypsinization. The purity of monocytes was greater than $85 \%$ as estimated by flow cytometry using antiCD14 IgG. The brief adherence step in the purification procedure did not affect monocyte CCR2 expression, and no difference in CCR2 expression was observed between purified monocytes and monocytes that were analyzed in freshly drawn blood using flow cytometry as described below.

For the in vivo experiments, tenth-generation homozygous LDL receptor-deficient mice in a C57BL/ 6 background were obtained from The Jackson Laboratories (Bar Harbor, Maine, USA). A breeding colony was generated, and 6- to 8-week-old female mice, weighing $17 \mathrm{~g}$, on average, were used. The animals were divided into two groups. Both groups received regular mouse chow (Harlan-Teklad, Madison, Wisconsin, USA), to which $0.075 \%$ cholesterol was added. The lowcholesterol addition did not significantly raise the plasma lipid level but maintained a level that was comparable between all animals. The animals were fed 3-4 $\mathrm{g}$ of food per mouse every day. In addition to the diet, for up to 8 weeks one group received BRL49653 at a concentration of $20 \mathrm{mg} / \mathrm{kg}$ body weight/day and the other a control solvent. The animals were weighed every 2 weeks and the drug dosage was adjusted accordingly. Blood samples were obtained retro-orbitally for determination of cholesterol and triglycerides. All animals received water ad libitum. The animal experiments were done according to NIH guidelines and were approved by University of California at San Diego Animal Subjects Committee. Mouse monocytes were isolated from 0.5-1 mL of whole blood, diluted with an equal volume of PBS containing $0.02 \%$ EDTA. Mononuclear leukocytes were separated by centrifugation through $3 \mathrm{~mL}$ of Histopaque 1077, and CCR2 protein was estimated using flow cytometry on gated CD80-positive monocytes, as described below. For the preparation of mRNA, mouse monocytes were further purified by plating for 1 hour in 10\% serum prepared from littermates with identical genetic background in $60-\mathrm{mm}$ dishes, as described above for human monocytes. 
For the various experiments, human monocytes were maintained in RPMI-1640 medium supplemented with $10 \%$ autologous serum. To avoid cell activation and differentiation, all incubations were carried out in 24-well plates that were coated with a hydrophobic surface to prevent adhesion (Sarstedt, Newton, North Carolina, USA). The incubation of THP-1 cells with lipoproteins or PPAR $\gamma$ ligands was carried out in RPMI-1640 containing 5\% lipoprotein-deficient serum. The final concentration of ethanol used to dissolve BRL49653 was $0.1 \%$ and did not affect monocyte viability or CCR 2 expression.

Preparation of lipoproteins. Human LDL was isolated from normolipidemic plasma by density ultracentrifugation (33). The LDL preparation was dialyzed against PBS with $0.3 \mathrm{mM}$ EDTA, and the protein concentration was determined (34). Before oxidation, EDTA was removed by extensive dialysis and OxLDL was prepared by incubation of LDL $(100 \mu \mathrm{g} / \mathrm{mL})$ with $10 \mu \mathrm{M} \mathrm{CuSO}_{4}$ in Ham's F-10 media for 18 hours at $37^{\circ} \mathrm{C}$. OxLDL contained $42 \mathrm{nmol}$ of thiobarbituric acid-reactive substances (TBARS) per milligram of protein (35). For mild oxidation of LDL (MM-LDL), EDTA-free LDL was incubated with $2 \mu \mathrm{M} \mathrm{CuSO}_{4}$ in Ham's F-10 media for 4 hours at $37^{\circ} \mathrm{C}$. MM-LDL contained $5 \mathrm{nmol}$ of TBARS per milligram of protein. To prevent further oxidation, $0.1 \mathrm{mM}$ EDTA was added and the lipoproteins were stored in the dark at $4^{\circ} \mathrm{C}$.

Isolation of lipids and apoB from lipoproteins and preparation of lipid microemulsions. ApoB was isolated from OxLDL by extracting the lipids with ice-cold chloroform/methanol (1:1 vol/vol) as described (36). The residual protein was washed with water and acetone, solubilized in octylglucoside (octylglucoside/protein, 30:1 wt/wt). The detergent was removed by dialysis against PBS, and the protein concentration was measured by the method of Lowry et al. (34).

Lipids from OxLDL were isolated as described (37). Briefly, $\mathrm{HCl}$ was added to LDL to a final concentration of $10 \mathrm{mM}$, the lipids were extracted with chloroform/methanol (1:1 vol/vol) and separated by centrifugation. The chloroform phase was removed, the solvent evaporated under $\mathrm{N}_{2}$, and the dried lipids were resuspended in $10 \mathrm{mM}$ Tris buffer containing $1 \mathrm{mM}$ EDTA and $150 \mathrm{mM} \mathrm{NaCl}, \mathrm{pH}$ 7.4. The lipid suspension was extruded eight to ten times through $0.1 \mu \mathrm{m}$ polycarbonate membranes under $\mathrm{N}_{2}$ at $37^{\circ} \mathrm{C}$ to form microemulsions with particle sizes of $80-120 \mathrm{~nm}$. For lipid labeling, $\mathrm{DiO}$ in an amount equal to $0.2 \%$ of the weight of the lipids was added to the chloroform phase before drying and resuspending the lipids in buffer for extrusion. For the preparation of microemulsions with PAPC or oxidized PAPC, the phospholipids were first mixed in chloroform with free cholesterol at a PAPC/cholesterol molar ratio of 2:1. The concentrations of lipid microemulsions were estimated by phosphorous assay (38). Linoleic acid and oxidized linoleic acid were used as solutions in ethanol, and 9-HODE and 13-HODE were dissolved in DMSO. For the oxi- dation, $1 \mathrm{mg}$ of PAPC or linoleic acid was dried on the surface of a 50-mL glass tube under $\mathrm{N}_{2}$ and exposed to air for 48 hours. The oxidation of the fatty acids proceeded to completion, which was confirmed by fattyacid analysis using gas chromatography (chromatograph from Varian, Walnut Creek, California, USA) as described (39) and by analysis of conjugated dienes as described (40).

Flow cytometry. Human and mouse mononuclear cells purified by centrifugation through Histopaque and THP-1 cells were washed twice with ice-cold PBS containing $0.1 \%$ BSA and $0.01 \%$ sodium azide (buffer A). The freshly isolated mononuclear cells $\left(10^{6}\right)$ or THP-1 cells $\left(2 \times 10^{5}\right)$ were resuspended in $100 \mu \mathrm{L}$ of buffer A. To block the Fc receptors, the cells were treated with 5 $\mu \mathrm{g}$ of nonspecific human or mouse IgG for 15 minutes at room temperature. Human and mouse monocytes were identified and gated by the expression of the surface markers CD14 (human monocytes) and CD80 (mouse monocytes) using the respective FITC-conjugated Ab's. To estimate monocyte CCR2 expression (CCR2 expression in the CD14- or CD80-positive cell population), cells were incubated with $0.25 \mu \mathrm{g}$ of phycoerythrin-conjugated mouse IgG against human CCR2 for 30 minutes at $4^{\circ} \mathrm{C}$. Mouse and human CCR2 share more than $80 \%$ homology in their amino-acid sequence, and our preliminary data showed that the polyclonal $\mathrm{Ab}$ reacted with both mouse and human CCR2. After labeling, the cells were washed twice with excess of ice-cold buffer A and analyzed by FACScan instrument using CELL QUEST software (Becton Dickinson, San Jose, California, USA). In control experiments phycoerythrin-conjugated nonspecific mouse IgG was used to measure nonspecific binding. The relative surface expression of CCR2 was estimated by subtracting the median fluorescence intensity of the control cells from that of cells labeled with anti-CCR2 Ab. This normalization method reduced variations between individual experiments. For the experiments with DiOlabeled OxLDL lipids, THP- 1 cells $\left(2 \times 10^{5}\right.$ cells $/ 100 \mu \mathrm{L}$ buffer A) were incubated with $0.5 \mu \mathrm{g}$ of DiO-labeled lipids from OxLDL for 1 hour at $4^{\circ} \mathrm{C}$ in the absence or presence of competitors. The assessment of DiO-labeled OxLDL-lipid binding using flow cytometry was essentially identical to the procedure described above.

${ }^{125}$ I-MCP-1 equilibrium-binding assay. Cells were washed twice with PBS, suspended in binding buffer consisting of RPMI-1640 without phenol red, $0.1 \%$ BSA, and $10 \mathrm{mM}$ HEPES ( $\mathrm{pH} 7.4$ ), and the binding analysis was carried out as described (25). Briefly, the cells $\left(10^{6}\right.$ in $200 \mu \mathrm{L}$ binding buffer) were incubated for 90 minutes at room temperature with $0.11 \mathrm{nM}^{125} \mathrm{I}-$ MCP-1 (specific activity, 2,200 Ci/mmol) in the absence or presence of $100 \mathrm{nM}$ unlabeled MCP-1. At the end of the incubation period, the cells were separated from the binding buffer by centrifugation through $500 \mu \mathrm{L}$ of dibutyl phthalate/dioctyl phthalate $(1: 1 \mathrm{vol} / \mathrm{vol})$, and the radioactivity of the cell pellet was counted. The specific ${ }^{125} \mathrm{I}-\mathrm{MCP}-1$ binding was 
a

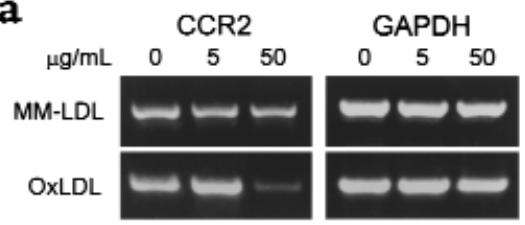

b

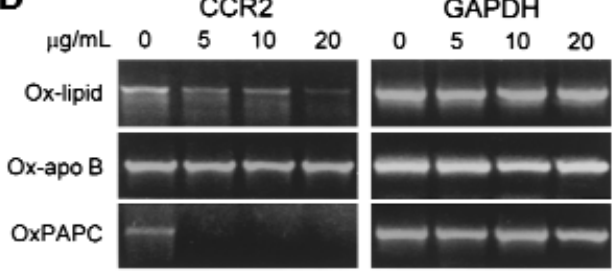

\section{Figure 1}

OxLDL lipids, but not apoB, reduce CCR2 transcripts in THP-1 cells. THP-1 cells were treated with modified intact LDL, with microemulsions of lipids from OxLDL, with reconstituted apoB from OxLDL, and with oxidized synthetic PAPC. The effect of the various treatments on CCR2 expression was determined by semiquantitative RT-PCR. GAPDH was analyzed under identical conditions and served as internal standard. (a) THP- 1 cells were incubated for 24 hours at $37^{\circ} \mathrm{C}$ with the indicated concentrations of either minimally modified LDL (MM$L D L$ ) or fully oxidized LDL (OxLDL). The LDL concentrations in the incubation medium are given in micrograms of protein per milliliter. (b) THP-1 cells were incubated with the indicated concentrations of either the isolated lipid (Ox-lipid) or the protein (Ox-apoB) moiety of OxLDL and with oxidized PAPC (OxPAPC). The lipid concentrations are expressed in terms of micrograms of phospholipid per milliliter.

determined by subtracting nonspecific binding estimated in the presence of $100 \mathrm{nM}$ unlabeled MCP-1 from total binding.

Quantification of CCR2 transcripts. Total RNA was isolated from 1-2 million monocytes by guanidinium thiocyanate-phenol-chloroform extraction (41). Transcripts from $0.5 \mu \mathrm{g}$ (circulating monocytes) or $2 \mu \mathrm{g}$ (THP-1 cells) of total RNA were reverse-transcribed by Superscript II (Life Technologies Inc., Gaithersburg, Maryland, USA) using oligo (dT) 10 primer (Roche Molecular Biochemicals, Indianapolis, Indiana, USA), and CCR2B expression was estimated by PCR, as described (42). For a more quantitative assessment of CCR2B transcripts, competitive RT-PCR analysis was performed. A competitor CDNA was constructed by deleting 185 internal nucleotides from CCR2B while retaining the recognition sites for the primers. Two primers with the sequences $5^{\prime}$-ATGCTGTCCACATCTCGTTCTCG$3^{\prime}$ (sense primer) and 5'-TTATAAACCAGCCGAGACTTCCTGC-3' (antisense primer) were used to amplify both full-length (1083 bp) and the truncated-competitor (898 bp) cDNA of CCR2B. The reverse-transcribed cDNA (5\% of the total) and diluted internal standard ranging from 200,000 to 2,000 molecules were combined and amplified in 30 cycles. PCR products were analyzed using agarose gel electrophoresis and densitometric scanning of the DNA bands (ImageQuant; Molecular Dynamics, Sunnyvale, California, USA). The densities of the bands of amplified CCR2B and truncated competitor were plotted, and the point at which both curves intersect indicates equal concentrations of both.

To ensure equal analysis conditions for both the semiquantitative and competitive RT-PCR, the intensity of all CCR2B bands were normalized to that of GAPDH, amplified and analyzed under identical conditions (22). Human GAPDH was amplified using the sense primer 5'-TCGGAGTCAACGGATTTGGTCGTA-3' and the antisense primer 5'-ATGGACTGTGGTCAGAGTCCTTC-3'. Mouse GAPDH was amplified using the sense and antisense primers 5'-TGCCATTTGCAGTGGCAAAGTGG-3' and 5'-TTGTCATGGATGACCTTGGCCAGG-3', respectively. To compensate for the relatively high expression of GAPDH, the reverse-transcribed cDNA was diluted to match the expression level of CCR2B. The concentration of the templates had been adjusted to give a linear relation between templates and products.

\section{Results}

We have reported previously that OxLDL significantly decreased the binding of ${ }^{125}$ I-MCP- 1 to THP-1 cells (25). The inhibition of CCR2-gene expression was particularly pronounced with OxLDL that was extensively modified (42 nmol TBARS/mg protein), whereas MM-LDL ( $5 \mathrm{nmol}$ TBARS/mg protein) displayed only modest effects (Figure 1a). To determine the mechanisms by which OxLDL inhibits CCR 2 expression, we incubated monocytic THP-1 cells for 24 hours with various concentrations of apoB purified from OxLDL and microemulsions prepared from lipids of OxLDL. The inhibitory activity on CCR2 transcription was clearly associated with the lipids from OxLDL, but not with the protein moiety (Figure 1b). Phospholipids make up about $25 \%$ of the total lipids of LDL and are a primary source of polyunsaturated fatty acids, which are highly susceptible to oxidation. Oxidized phospholipids, as contained in OxLDL, have been shown to have a number of biological effects in cultured cells $(43,44)$. To explore if they can also mediate the reduction of CCR2 expression, we oxidized synthetic PAPC and incubated THP1 cells with microemulsions prepared from it. As shown in Figure 1b, oxidized PAPC inhibited CCR2 transcription quite efficiently. Estimation of CCR2 surface expression using flow cytometry gave similar results. As expected, OxLDL and the lipids prepared from it reduced CCR2 protein in a dose-dependent manner (Figure 2a). In contrast, reconstituted apoB isolated from OxLDL showed no inhibitory activity (Figure 2a). The inhibition of CCR2 expression by the OxLDL lipids including phospholipids was strictly oxidation dependent, and only oxidized PAPC reduced CCR2 protein, whereas no changes were observed with native PAPC (Figure 2b). Lysophosphatidylcholine, another component of OxLDL, had no effect on CCR 2 expression at concentrations up to $20 \mu \mathrm{g} / \mathrm{mL}$ (data not shown). Oxidized lipids may exert certain cytotoxic effects and were therefore used only 

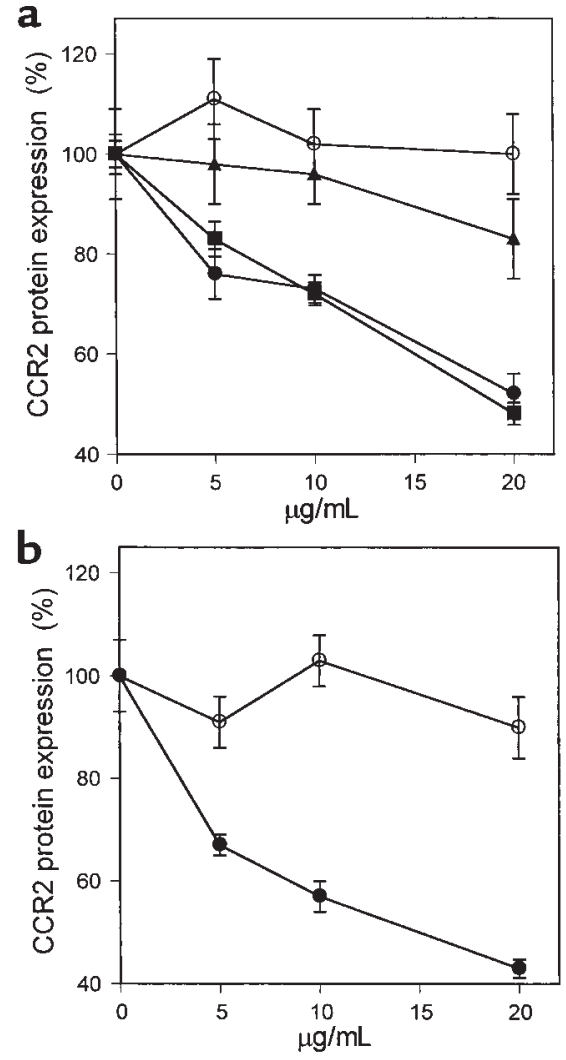

Figure 2

Effect of OxLDL and oxidized phospholipids on expression of CCR2 protein. THP-1 cells were treated with OxLDL and the various protein and lipid fractions prepared from it as described in Figure 1. CCR2 protein was estimated by flow cytometry using a phycoerythrin-labeled mouse anti-human CCR2 Ab. Nonspecific fluorescence was obtained by labeling the cells with phycoerythrin-conjugated mouse isotype $\lg G$. The median CCR2-specific fluorescence is shown as percentage of protein expression relative to that of untreated THP1 control cells (100\%). The concentrations of the lipoproteins are given in micrograms of protein per milliliter and concentrations of the lipids in micrograms of phospholipids per milliliter. (a) THP-1 cells incubated with the indicated concentrations of MM-LDL (filled triangles), OxLDL (filled squares), microemulsion of lipids from OxLDL (filled circles), and reconstituted apoB from OxLDL (open circles). (b) THP-1 cells treated with oxidized (filled circles) and native (open circles) PAPC. The data represent the means \pm SD of three independent experiments.

at low concentrations. The possible cytotoxicity of any of the materials at the concentrations used was ruled out by flow cytometry using propidium iodide staining. Under our experimental conditions, the percentage of cells that stained with propidium iodide was less than $3 \%$, essentially excluding cellular damage as cause for the reduction of CCR2 expression.

To test if the inhibitory effect of OxLDL was mediated by receptor-dependent transmembrane signaling and to identify the receptor involved in this process, we performed competition-binding experiments. As shown in Figure 3, the binding of DiO-labeled OxLDL lipids to THP- 1 cells was specific and was inhibited by an excess of unlabeled lipids from OxLDL, as well as by microemulsions of oxidized PAPC. A similar inhibition in the binding was also seen with apoB from OxLDL (Figure 3), but in contrast to intact OxLDL or lipids prepared from it, it had no effect on CCR2 expression (Figure 2). These results indicated that OxLDL regulates monocyte CCR2-gene expression by pathways other then those that require ligand-induced transmembrane signaling.

Next we determined if oxidized lipids can stimulate intracellular signaling pathways and whether receptormediated internalization was necessary. In a recent study we have demonstrated that CD36 binds both the protein and lipid moieties of OxLDL with high affinity (45). To test for a specific involvement of CD36 in the delivery of oxidized lipids, we incubated THP-1 cells with DiOlabeled OxLDL lipids in the presence of a neutralizing mouse anti-human CD36 Ab, OKM5. Consistent with an important function for CD36, OKM5 substantially reduced the binding of the oxidized lipids (Figure 3 ). The functional blocking of CD36 also prevented the negative regulatory effects on CCR2 expression. Monocyte CCR2 expression, reduced by OxLDL or oxidized lipids, was completely restored in the presence of OKM5 (Figure 4). Prolonged exposure of monocytes to high concentrations of OxLDL potentially can induce differentiation and downregulation of CCR 2 expression. To ensure that the ensuing inhibition of CCR2 expression was directly related to OxLDL and not to differentiation, we used exceptionally low concentrations $(10 \mu \mathrm{g} / \mathrm{mL})$ of OxLDL or oxidized lipids in these experiments. No morpholog-

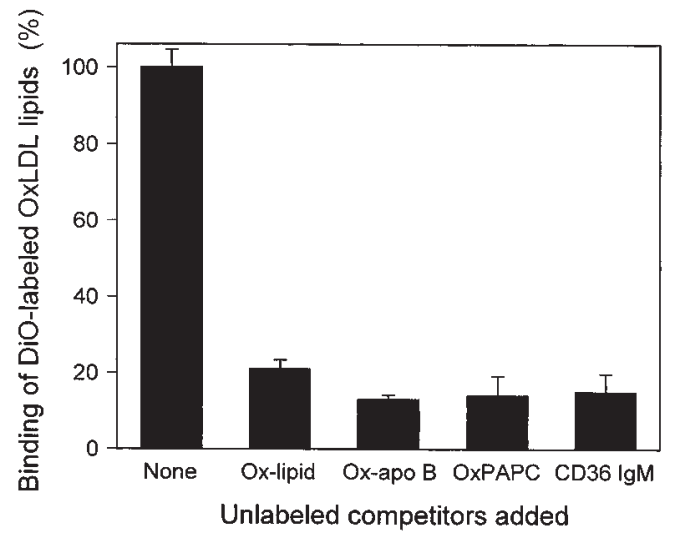

Figure 3

Inhibition of THP-1-cell binding of DiO-labeled OxLDL lipids by various competitors. THP- 1 cells were incubated for 1 hour at $4^{\circ} \mathrm{C}$ with microemulsions of DiO-lipids from OxLDL $(0.5 \mu \mathrm{g}$ of phospholipids per milliliter) in the absence (None) or presence of unlabeled lipids from OxLDL (Ox-lipid) at $20 \mu \mathrm{g}$ phospholipids/mL, apoB from OxLDL (Ox-apoB) at $20 \mu \mathrm{g} / \mathrm{mL}$, oxidized synthetic PAPC (OxPAPC) at $20 \mu \mathrm{g} / \mathrm{mL}$ phospholipids, and $10 \mu \mathrm{g} / \mathrm{mL}$ neutralizing mouse anti-human CD36 IgM, OKM5. The effect of the various competitors on the binding of DiO-OxLDL lipids was assessed by flow cytometry. All data are expressed as percentage of binding relative to that seen in the absence of competitor (100\%) and represent the means \pm SD of three independent experiments. The reductions in binding by the various competitors are statistically significant $(P<0.001$, unpaired Student's $t$ test $)$. 


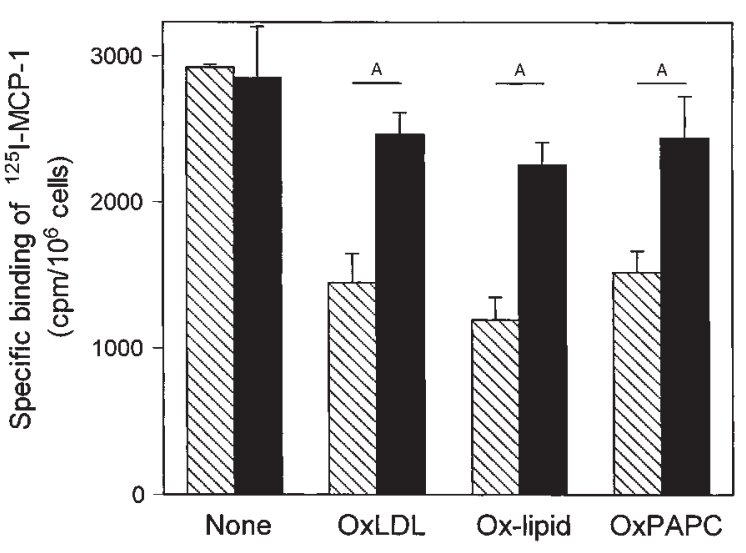

Figure 4

Neutralizing anti-human CD36 IgM, OKM5, blocks the negative regulatory effects of OxLDL and oxidized lipids on CCR2 expression. THP1 cells were incubated for 24 hours with $10 \mu \mathrm{g} / \mathrm{mL}$ of OxLDL (OxLDL), microemulsions of lipids from OxLDL (Ox-lipid), and microemulsions of oxidized PAPC (OxPAPC) at concentrations of $10 \mu \mathrm{g}$ phospholipid/ $\mathrm{mL}$ in the absence (hatched bars) or presence (solid bars) of neutralizing anti-human CD36 IgM at $10 \mu \mathrm{g} / \mathrm{mL}$. After 24 hours, the cells were harvested by centrifugation and CCR 2 expression was estimated by ${ }^{125} \mathrm{I}-\mathrm{MCP}-1$-binding analysis. Binding of ${ }^{125} \mathrm{I}-\mathrm{MCP}-1$ to untreated control cells was, on average, $3.8 \mathrm{fmol} / 10^{6}$ cells, which was not affected by the Ab. All data represent the mean \pm SD of three independent experiments. ${ }^{A} P<0.01$ (unpaired Student's $t$ test).

ical changes characteristic for differentiation were detectable, and the inhibitory effects were significant even at the low agonist concentrations.

It has been shown previously that oxidized metabolites of linoleic or arachidonic acid, which are present in OxLDL, are ligands of PPAR $\gamma(46,47)$. To investigate whether CCR 2 expression is regulated by PPAR $\gamma$ dependent mechanisms, we incubated THP-1 cells with oxidized linoleic acid. As shown in Figure 5, oxidized linoleic acid and metabolites derived from it, including 9-HODE and 13-HODE, inhibited CCR2gene expression and reduced both CCR2 protein and CCR2 mRNA. In contrast, nonoxidized linoleic acid had no effects on CCR2 expression. These results suggested that PPAR $\gamma$ might be a negative regulator for CCR2 expression. To more directly establish a role of PPAR $\gamma$ in CCR2 expression, we incubated THP- 1 cells with $1 \mu \mathrm{M}$ BRL49653 and measured the effect on the functional expression of CCR2 by ${ }^{125} \mathrm{I}-$ MCP-1-binding analysis. BRL49653 (rosiglitazone) is a well-characterized synthetic ligand for $\operatorname{PPAR} \gamma(28$, 48). As shown in Figure 6, BRL49653 reduced CCR2 surface expression by about $50-60 \%(P<0.001)$ compared with untreated control cells. We have reported previously that proinflammatory cytokines also reduce CCR2 expression (42) and have therefore included TNF- $\alpha$ as positive control. The change in CCR 2 protein induced by BRL49653 was consistent with the change in CCR 2 mRNA, which decreased about 2.3 -fold after 72 hours, as determined by competitive RT-PCR (Figure 7). A similar inhibition in CCR2 expression was also observed with freshly iso- lated human monocytes that were treated ex vivo with the PPAR $\gamma$ ligand. Human monocyte CCR2 mRNA was reduced about 3.7-fold after 72 hours of incubation with $1 \mu \mathrm{M}$ BRL49653 compared with the controls that were maintained in the absence of the drug (Figure 8). The level of CCR 2 mRNA of control monocytes cultured under identical conditions remained unchanged, and no difference in CCR2 transcripts was found between freshly isolated monocytes and monocytes that were cultured for 72 hours in the absence of the drug. Because CCR2 expression is sensitive to cell activation and differentiation (42), these results suggest that the control monocytes did not become activated during isolation or subsequent incubation. The effects on CCR 2 expression are, therefore, directly related to the drug treatment. The level of protein expression, determined by flow cytometry, paralleled that of the mRNA and was reduced by about $39 \pm 15 \%$ and $57 \pm 12 \%$ after 48 and 72 hours, respectively $(n=3)$.

PPAR $\gamma$ activation by natural or synthetic ligands may promote monocyte/macrophage differentiation (30), which could cause a downregulation of CCR 2 expression (42). To prevent differentiation, which was examined by estimating the expression of the differentiation-dependent surface marker CD14, we selected the

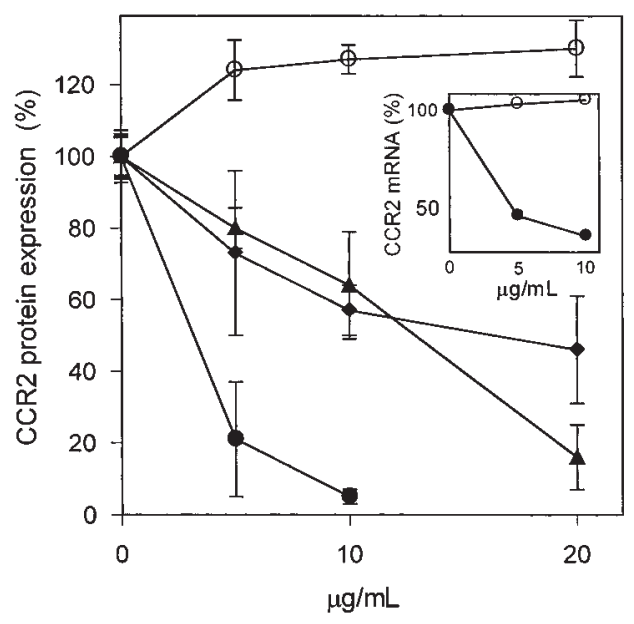

Figure 5

Reduction of CCR2 expression by oxidized linoleic acid. THP-1 cells were incubated for 24 hours with the indicated concentrations of native linoleic acid (open circles), oxidized linoleic acid (filled circles), 9-HODE (filled triangles), and 13-HODE (filled diamonds). Due to cytotoxic effects observed at high concentrations, the final concentration of oxidized linoleic acid was limited to $10 \mu \mathrm{g} / \mathrm{mL}$. CCR2 protein was estimated by flow cytometry using phycoerythrin-conjugated anti-CCR2 IgG. Nonspecific fluorescence, obtained by labeling of the cells with phycoerythrin-conjugated human isotype IgG, was subtracted, and the median CCR2-specific fluorescence is shown as percentage of protein expression. The values represent the means \pm SD of three independent experiments. Inset: The cells were incubated for 24 hours with the indicated concentrations of native (open circles) or oxidized (filled circles) linoleic acid. CCR2 mRNA was estimated by semiquantitative RT-PCR and normalized to GAPDH mRNA estimated under identical conditions. 


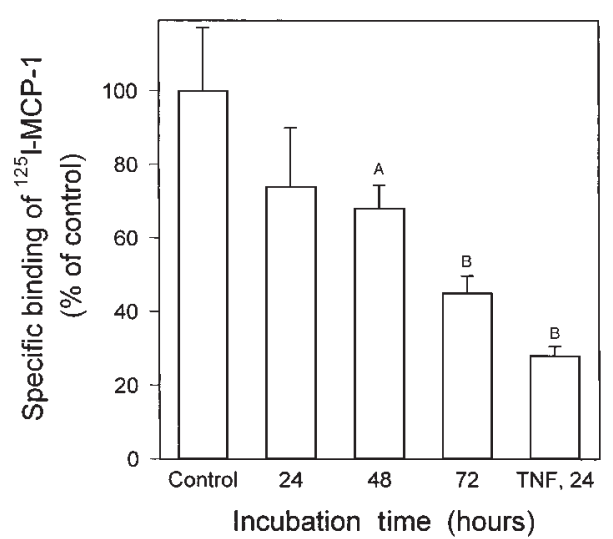

\section{Figure 6}

Activation of PPAR $\gamma$ reduced the expression of functional CCR2. THP-1 cells were incubated for the indicated time periods with $1 \mu \mathrm{M}$ BRL49653, and the effect of the synthetic PPAR $\gamma$ ligand on CCR2 expression was estimated using ${ }^{125}$ I-MCP-1-binding assays. Cells that were kept for 72 hours without any additions were used as control. Data are expressed as percentage of specific binding relative to that of control cells (100\%), which bound $2.4 \pm 0.4 \mathrm{fmol}{ }^{125} \mathrm{I}-\mathrm{MCP}-1$ per $10^{6}$ cells. As a positive control, cells were treated for 24 hours with $10 \mathrm{ng} / \mathrm{mL}$ TNF- $\alpha$ (TNF, 24), which is known to decrease CCR2 expression (42). All data represent the mean \pm SD of three independent experiments. ${ }^{A} P<0.05$; ${ }^{B} P<0.001$ (Mann Whitney $U$ test).

lowest effective concentration of BRL49653 (1 $\mu \mathrm{M})$. At this concentration, the drug did not induce expression of CD14 or any morphological change characteristic for differentiation. Similarly, neither oxidized linoleic acid nor PAPC at concentrations used in the experiments affected CD14 expression.

To demonstrate a physiological role of PPAR $\gamma$ activation in regard to CCR2 expression in vivo, LDL receptor-negative mice were treated with BRL49653 $(20 \mathrm{mg} / \mathrm{kg}$ body weight/day) for 2 and 8 weeks. The plasma concentration of the drug was on average 5.1 $\pm 0.6 \mu \mathrm{g} / \mathrm{mL}$. Plasma lipid levels were not affected, and total cholesterol $(292.5 \pm 49.2 \mathrm{mg} / \mathrm{dL})$ as well as triglycerides $(77.8 \pm 13.3 \mathrm{mg} / \mathrm{dL})$ were not significantly different from those of the control animals that did not receive the drug treatment. In contrast, CCR2 protein expression was on average 2.9- and 2.6fold lower in circulating monocytes from animals treated for 2 and 8 weeks, respectively, compared with monocytes from the control group (Figure 9a). The difference between the 2- and 8-week treatment was not statistically significant. Consistent with our observations from the in vitro studies that suggested that BRL49653 controls CCR2-gene transcription, mRNA levels were reduced similarly by the treatment (Figure 9b). Together our data provide compelling evidence that activation of PPAR $\gamma$ causes a reduction in monocyte CCR 2 expression both in vitro and in vivo.

\section{Discussion}

Our data demonstrate that OxLDL effectively downregulates the expression of CCR2 in monocytes. OxLDL is present in atherosclerotic lesions of all stages and is thought to be critically involved in the pathogenesis of atherosclerosis. Oxidative modification of LDL stimulates its uptake by macrophages via scavenger receptors, which results in the formation of foam cells, recognized as the initial stage of a developing atherosclerotic lesion (49). OxLDL exhibits many other potentially proatherogenic properties. It stimulates the expression of MCP-1, adhesion molecules, and cytokines in cells of the vessel wall, including endothelial cells and smooth muscle cells, which promote the recruitment of monocytes $(2,7)$. OxLDL affects gene expression in leukocytes also, and in a previous study we have demonstrated that exposure of monocytes to OxLDL reduces CCR2 expression, causes a rapid loss of functional CCR2 protein, and inhibits the physiological response of monocytes to MCP-1 (25).

In this study we examined the mechanisms by which OxLDL regulates monocyte CCR 2 expression. We now provide evidence that receptor-mediated uptake of OxLDL induces intracellular signaling and causes an inhibition of CCR2B expression by mechanisms that may involve the activation of the ligand-dependent transcription factor PPAR $\gamma$. Our results demonstrate that the effect of OxLDL on monocyte CCR 2 expression is associated with the lipid moiety of the modified lipoprotein, which most likely contains the ligands for PPAR $\gamma$ such as oxidized polyunsaturated fatty acids. Microemulsions prepared from lipids extracted from OxLDL reduced CCR 2 expression in THP-1 cells as effectively as did the intact particle. Similar inhibition was seen also with an oxidized synthetic phospholipid, namely PAPC. In contrast, reconstituted apoB from OxLDL after exhaustive extraction of the lipids had no effect on monocyte CCR2 expression, although it com-

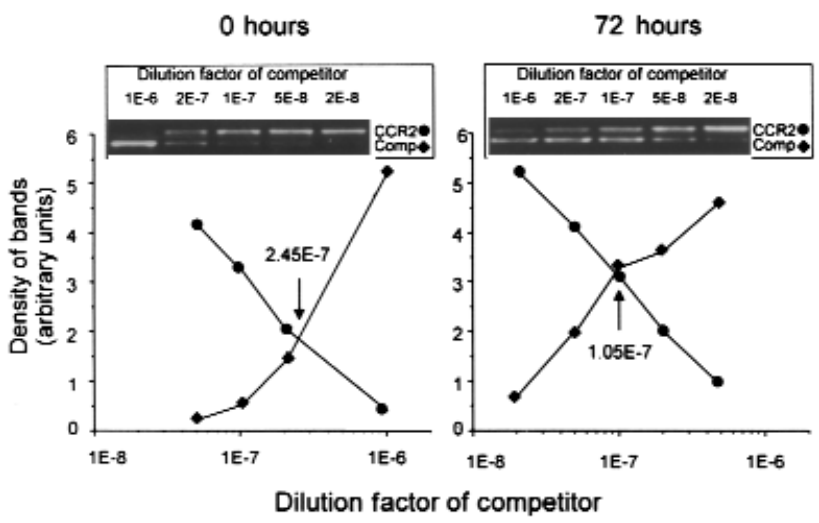

\section{Figure 7}

PPAR $\gamma$-mediated reduction of CCR 2 mRNA. THP- 1 cells $\left(10^{6}\right)$ were incubated for 72 hours in the absence ( 0 hours) or presence (72 hours) of $1 \mu \mathrm{M}$ BRL49653, as described in Figure 6, and CCR2 transcripts were estimated using competitive RT-PCR. The competitor was added at final dilutions indicated in the graphs. At a dilution of $10^{-8}, 2,000$ molecules of competitor were present. The point at which both curves intersect indicates equal concentration of CCR2 and competitor templates. GAPDH was analyzed by semiquantitative RT-PCR to ensure that equal amounts of RNA were used. 

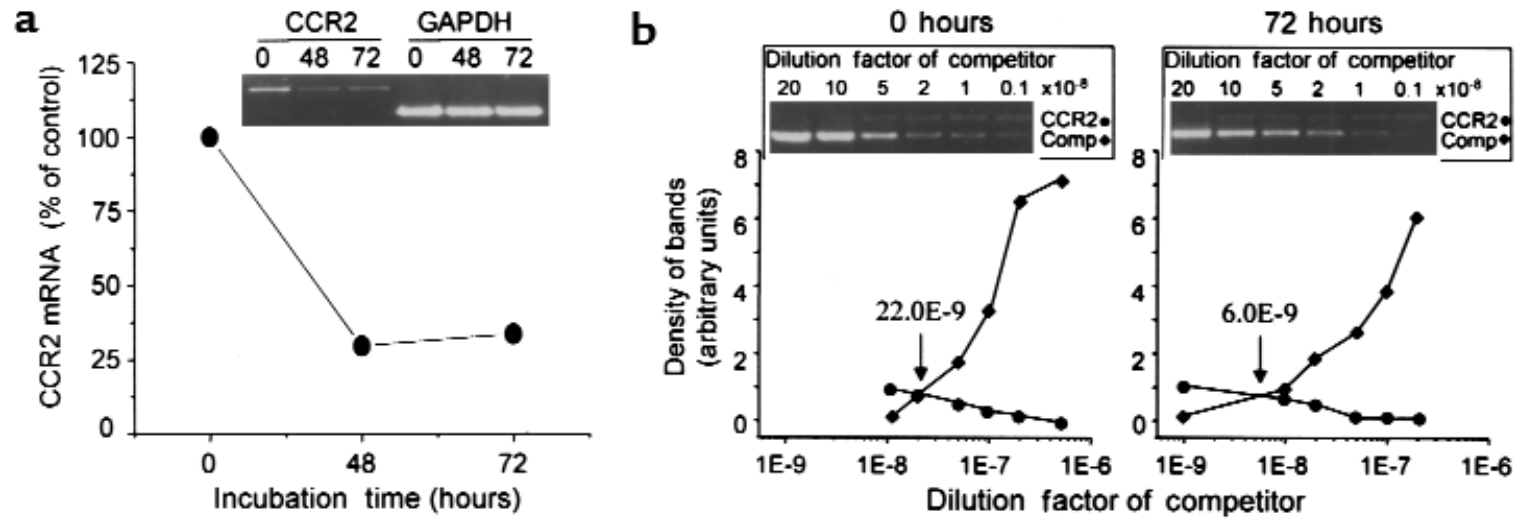

Figure 8

Effect of BRL49653 on CCR2 expression in freshly isolated human monocytes. Freshly isolated human monocytes were cultured in the absence or presence of $1 \mu \mathrm{M}$ BRL49653 for the indicated time periods. Total RNA was isolated from $10^{6}$ cells, and $0.5 \mu \mathrm{g}$ was used for analysis of CCR2 transcripts. (a) The time course of the BRL49653-induced reduction of CCR2 mRNA was analyzed by semiquantitative RT-PCR. As an internal standard, GAPDH was analyzed under identical conditions. (b) CCR2 transcripts of cells cultured for 72 hours in the absence (0 hours) or presence (72 hours) of $1 \mu \mathrm{M}$ BRL49653 were further analyzed using competitive RT-PCR as described in Figure 7 . A dilution factor of $10^{-8}$ represents 2,000 molecules of competitor templates in the reaction mix. The point at which both curves intersect indicates that both CCR2 and competitor templates were present at equal concentrations.

peted for the binding of oxidized lipids.

PPAR $\gamma$ is a member of the nuclear receptor superfamily that also includes PPAR $\alpha$ and PPAR $\delta$. Although they all are activated by a diverse group of lipids, including long-chain fatty acids and metabolites such as prostaglandins, certain ligand specificity has been observed. Both PPAR $\alpha$ and PPAR $\delta$ recognize polyunsaturated fatty acids, including linoleic acid, which in its nonoxidized form is a poor activator for PPAR $\gamma(50)$. However, oxidized metabolites of linoleic and arachidonic acid, including 9-HODE and 13HODE, are very effective PPAR $\gamma$ activators $(46,47)$. Consistent with an involvement of PPAR $\gamma$, oxidized linoleic acid, 9-HODE and 13-HODE, reduced very potently monocyte CCR2 expression (Figure 5). In contrast, native linoleic acid without oxidative modifications had no effect. Linoleic acid is the major polyunsaturated fatty acid in LDL and therefore the predominant target for peroxidation. Although the mechanisms by which LDL is oxidized in vivo are not clearly established, there is substantial evidence that cellular 15-lipoxygenase contributes to the formation of OxLDL $(51,52)$. The enzymatic reaction results in regiospecific oxidation of polyunsaturated fatty acids to produce the known ligands for PPAR $\gamma$ in OxLDL (53). Our results with oxidized linoleic acid are consistent with a major role for PPAR $\gamma$ in the OxLDLinduced reduction of CCR 2 expression.

More direct evidence for a causative role of PPAR $\gamma$ was obtained with BRL49653, a synthetic ligand that specifically activates PPAR $\gamma(28)$. PPAR $\gamma$ is expressed in human peripheral blood monocytes and in monocytic cell lines (30). Activation of PPAR $\gamma$ with BRL49653 decreased in a time-dependent fashion CCR2 expression in both cultured monocytic THP-1 cells and freshly isolated human monocytes ex vivo. A significant decrease (30\%) was already observed after 24 hours of treatment, and after 72 hours functional CCR 2 protein was reduced by about $60 \%$. At this expression level, the chemotactic response of monocytes to MCP-1 is greatly diminished (25). The continued reduction of CCR2 seen at later time points is probably due to OxLDL-induced stimulation of PPAR $\gamma$ expression (30).
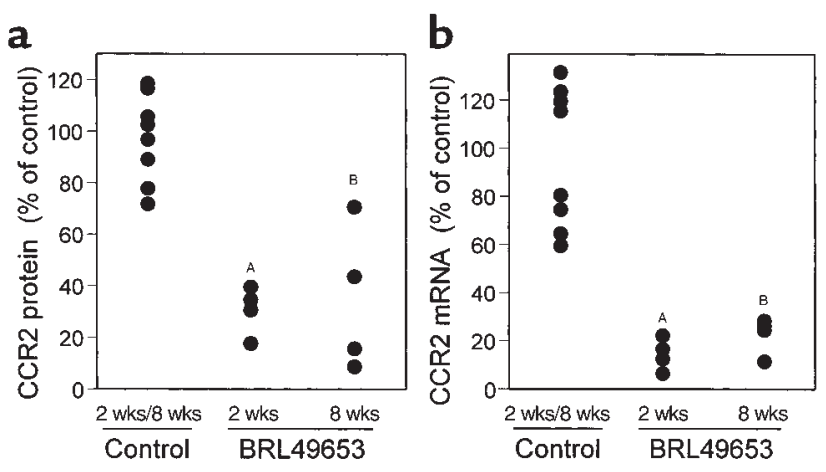

Figure 9

Reduction of CCR2 expression in circulating monocytes by BRL49653 in vivo. LDL receptor-deficient mice were placed for 2 and 8 weeks on BRL49653 (20 mg/kg body weight), and the effect on CCR2 expression in circulating monocytes was determined. (a) Analysis of CCR2 protein by flow cytometry. Mononuclear leukocytes were isolated from untreated control $(n=8)$ and BRL49653-treated $(n=4)$ animals. The monocyte population was identified with anti-CD80 Ab, stained with phycoerythrin-conjugated anti-CCR2 IgG, and analyzed by flow cytometry. In preliminary experiments it was established that the antihuman CCR2 IgG crossreacted with mouse CCR2. Phycoerythrinlabeled non-specific IgG was used to estimate background fluorescence. (b) Analysis of CCR2 transcripts. The monocytes were purified from mononuclear leukocytes by plating. The levels of CCR2 mRNA in circulating monocytes from control and treated animals were estimated by semiquantitative RT-PCR using $0.5 \mu \mathrm{g}$ of total RNA. All values shown are normalized to GAPDH. $P$ values were estimated using the unpaired Student's $t$ test. ${ }^{A} P<0.01 ;{ }^{B} P<0.05$. 
Some recently published results suggested that BRL49653 induced CCR2 expression in THP-1 cells, which conflicts with our observation (54). In that report the THP-1 cells used did not show any MCP1-mediated chemotactic responses, indicating that a partially differentiated, heterogeneous cell culture with very low basal expression of CCR2 may have been used. From previous studies we know that culture conditions greatly affect the maturation level of THP1 cells and CCR 2 expression. THP- 1 cells maintained under conditions that are routinely applied in our experiments expressed CCR 2 at levels that were similar to that of freshly isolated human monocytes, and the cells showed comparable chemotactic responses to MCP-1 $(25,42)$. To confirm our data on monocytic THP-1 cells, we included freshly isolated human monocytes in our study. In both cell types PPAR $\gamma$ activation caused a reduction in CCR2 expression.

To further demonstrate the involvement of PPAR $\gamma$ in the regulation of monocyte CCR2 expression and to establish physiological relevance in vivo, we examined the effect of BRL49653 on monocyte CCR2 expression in mice. In all treated animals, the PPAR $\gamma$ ligand substantially reduced both CCR2 protein and mRNA in circulating monocytes. These results confirm the findings of the in vitro studies with cell cultures or freshly isolated human monocytes and are consistent with recent reports demonstrating that PPAR $\gamma$ is a regulator of monocyte/macrophage gene expression $(32,46)$.

Lipids from OxLDL do not diffuse freely across the cell membrane, and the delivery of PPAR $\gamma$ ligands involves particle internalization that is mediated by scavenger receptors. CD36 is one of the major scavenger receptors responsible for binding and internalization of OxLDL by macrophages $(45,55,56)$. Consistent with an important role in monocyte function, our data indicated that CD36 is the primary monocyte scavenger receptor responsible for most of the binding of OxLDL and delivery of PPAR $\gamma$ ligands. In addition to its role as a scavenger receptor, there is evidence that CD36 can also function as a signal-transduction molecule (57). However, our data do not support such a function in regard to the inhibition of CCR2 expression by OxLDL. Although both moieties are ligands for CD36 and appear to bind to the same receptor site, only the oxidized lipids inhibited monocyte CCR2 expression, whereas apoB from OxLDL had no effect at all.

In summary, the data presented in this work implicate PPAR $\gamma$ in the inhibition of CCR 2 expression by OxLDL. The regulation of chemokine-receptor expression may be a crucial mechanism to control monocyte responses to chemokines. Any disturbances of the delicate balance that governs a tolerable immune response may cause pathological conditions such as atherosclerosis. High plasma levels of LDL increase CCR2 expression and the chemotactic response of monocytes to MCP-1 $(22,25)$. In contrast, OxLDL reduces CCR 2 expression, which may block reverse transmigration and retain monocytes at sites of inflammation such as the atherosclerotic lesion. This may promote excessive monocyte accumulation, foam cell formation, and may accelerate atherogenesis.

\section{Acknowledgments}

This work was supported by NIH grant HL-56989 (Specialized Center of Research in Molecular Medicine and Atherosclerosis, La Jolla, California, USA) and by a grant from the American Heart Association (to O. Quehenberger). K.H. Han was supported by a fellowship from the American Heart Association, and M.K. Chang was supported by a fellowship from the Tobacco-Related Disease Research Program, California. O. Quehenberger is an Established Investigator of the American Heart Association.

1. Gerrity, R.G. 1981. The role of the monocyte in atherogenesis. I. Transition of blood-borne monocytes into foam cells in fatty lesions. Am. J. Pathol. 103:181-190.

2. Valente, A.J., Rozek, M.M., Sprague, E.A., and Schwartz, C.J. 1992. Mechanisms in intimal monocyte-macrophage recruitment. A special role for monocyte chemotactic protein-1. Circulation. 86:III20-III25.

3. Rollins, B.J., Yoshimura, T., Leonard, E.J., and Pober, J.S. 1990. Cytokineactivated human endothelial cells synthesize and secrete a monocyte chemoattractant, MCP-1/JE. Am. J. Pathol. 136:1229-1233.

4. Shyy, Y.J., et al. 1993. Human monocyte colony-stimulating factor stimulates the gene expression of monocyte chemotactic protein-1 and increases the adhesion of monocytes to endothelial monolayers. J. Clin. Invest. 92:1745-1751.

5. Brown, Z., et al. 1994. Chemokine gene expression and secretion by cytokine-activated human microvascular endothelial cells: differential regulation of monocyte chemoattractant protein- 1 and interleukin- 8 in response to interferon-gamma. Am. J. Pathol. 145:913-921.

6. Wenzel, U.O., et al. 1995. Thrombin regulates expression of monocyte chemoattractant protein-1 in vascular smooth muscle cells. Circ. Res. 77:503-509.

7. Cushing, S.D., et al. 1990. Minimally modified low density lipoprotein induces monocyte chemotactic protein 1 in human endothelial cells and smooth muscle cells. Proc. Natl. Acad. Sci. USA. 87:5134-5138.

8. Nelken, N.A., Coughlin, S.R., Gordon, D., and Wilcox, J.N. 1991. Monocyte chemoattractant protein-1 in human atheromatous plaques. J. Clin. Invest. 88:1121-1127.

9. Yla-Herttuala, S., et al. 1991. Expression of monocyte chemoattractant protein 1 in macrophage- rich areas of human and rabbit atherosclerotic lesions. Proc. Natl. Acad. Sci. USA. 88:5252-5256.

10. Gu, L., et al. 1998. Absence of monocyte chemoattractant protein-1 reduces atherosclerosis in low density lipoprotein receptor-deficient mice. Mol. Cell. 2:275-281.

11. Gosling, J., et al. 1999. MCP-1 deficiency reduces susceptibility to atherosclerosis in mice that overexpress human apolipoprotein B. J. Clin. Invest. 103:773-778.

12. Aiello, R.J., et al. 1999. Monocyte chemoattractant protein-1 accelerates atherosclerosis in apolipoprotein E-deficient mice. Arterioscler. Thromb. Vasc. Biol. 19:1518-1525.

13. Rollins, B.J., Morrison, E.D., and Stiles, C.D. 1988. Cloning and expression of JE, a gene inducible by platelet- derived growth factor and whose product has cytokine-like properties. Proc. Natl. Acad. Sci. USA. 85:3738-3742.

14. Yoshimura, T., et al. 1989. Purification and amino acid analysis of two human glioma-derived monocyte chemoattractants. J. Exp. Med. 169:1449-1459.

15. Charo, I.F., et al. 1994. Molecular cloning and functional expression of two monocyte chemoattractant protein 1 receptors reveals alternative splicing of the carboxyl-terminal tails. Proc. Natl. Acad. Sci. USA. 91:2752-2756.

16. Mackay, C.R. 1996. Chemokine receptors and T cell chemotaxis. J. Exp. Med. 184:799-802.

17. Monteclaro, F.S., and Charo, I.F. 1997. The amino-terminal domain of CCR2 is both necessary and sufficient for high affinity binding of monocyte chemoattractant protein 1 . Receptor activation by a pseudo-tethered ligand. J. Biol. Chem. 272:23186-23190.

18. Han, K.H., Green, S.R., Tangirala, R.K., Tanaka, S., and Quehenberger, O. 1999. Role of the first extracellular loop in the functional activation 
of CCR2: the first extracellular loop contains distinct domains necessary for both agonist binding and transmembrane signaling. J. Biol. Chem. 274:32055-32062.

19. Kuziel, W.A., et al. 1997. Severe reduction in leukocyte adhesion and monocyte extravasation in mice deficient in CC chemokine receptor 2. Proc. Natl. Acad. Sci. USA. 94:12053-12058.

20. Boring, L., et al. 1997. Impaired monocyte migration and reduced type 1 (Th1) cytokine responses in C-C chemokine receptor 2 knockout mice. J. Clin. Invest. 100:2552-2561.

21. Boring, L., Gosling, J., Cleary, M., and Charo, I.F. 1998. Decreased lesion formation in CCR2 $2^{-/}$mice reveals a role for chemokines in the initiation of atherosclerosis. Nature. 394:894-897.

22. Han, K.H., Han, K.O., Green, S.R., and Quehenberger, O. 1999. Expression of the monocyte chemoattractant protein-1 receptor CCR2 is increased in hypercholesterolemia: differential effects of plasma lipoproteins on monocyte function. J. Lipid Res. 40:1053-1063.

23. Steinberg, D., Parthasarathy, S., Carew, T.E., Khoo, J.C., and Witztum, J.L. 1989. Beyond cholesterol. Modifications of low-density lipoprotein that increase its atherogenicity. N. Engl. J. Med. 320:915-924.

24. Steinberg, D. 1997. Low density lipoprotein oxidation and its pathobiological significance. J. Biol. Chem. 272:20963-20966.

25. Han, K.H., Tangirala, R.K., Green, S.R., and Quehenberger, O. 1998 Chemokine receptor CCR2 expression and monocyte chemoattractant protein-1-mediated chemotaxis in human monocytes. A regulatory role for plasma LDL. Arterioscler. Thromb. Vasc. Biol. 18:1983-1991.

26. Tontonoz, P., Hu, E., Graves, R.A., Budavari, A.I., and Spiegelman, B.M. 1994. mPPAR gamma 2: tissue-specific regulator of an adipocyte enhancer. Genes Dev. 8:1224-1234.

27. Chawla, A., Schwarz, E.J., Dimaculangan, D.D., and Lazar, M.A. 1994. Peroxisome proliferator-activated receptor (PPAR) gamma: adipose-predominant expression and induction early in adipocyte differentiation. Endocrinology. 135:798-800.

28. Lehmann, J.M., et al. 1995. An antidiabetic thiazolidinedione is a high affinity ligand for peroxisome proliferator-activated receptor gamma (PPAR gamma). J. Biol. Chem. 270:12953-12956.

29. Willson, T.M., et al. 1996. The structure-activity relationship between peroxisome proliferator-activated receptor gamma agonism and the antihyperglycemic activity of thiazolidinediones. J. Med. Chem. 39:665-668.

30. Tontonoz, P., Nagy, L., Alvarez, J.G., Thomazy, V.A., and Evans, R.M. 1998. PPARgamma promotes monocyte/macrophage differentiation and uptake of oxidized LDL. Cell. 93:241-252.

31. Ricote, M., et al. 1998. Expression of the peroxisome proliferator-activated receptor gamma (PPARgamma) in human atherosclerosis and regulation in macrophages by colony stimulating factors and oxidized low density lipoprotein. Proc. Natl. Acad. Sci. USA. 95:7614-7619.

32. Ricote, M., Huang, J.T., Welch, J.S., and Glass, C.K. 1999. The peroxisome proliferator-activated receptor(PPARgamma) as a regulator of monocyte/macrophage function. J. Leukoc. Biol. 66:733-739.

33. Havel, R.J., Eder, H.A., and Bragdon, J.H. 1955. The distribution and chemical composition of ultracentrifugally separated lipoproteins in human serum. J. Clin. Invest. 34:1345-1353.

34. Lowry, O.H., Rosebrough, N.J., Farr, A.L., and Randall, R.J. 1951. Protein measurement with the folin phenol reagent. J. Biol. Chem. 193:265-275.

35. Conti, M., Morand, P.C., Levillain, P., and Lemonnier, A. 1991. Improved fluorometric determination of malonaldehyde. Clin. Chem. 37:1273-1275.

36. Parthasarathy, S., Fong, L.G., Otero, D., and Steinberg, D. 1987. Recognition of solubilized apoproteins from delipidated, oxidized low density lipoprotein (LDL) by the acetyl-LDL receptor. Proc. Natl. Acad. Sci. USA. 84:537-540.

37. Terpstra, V., Bird, D.A., and Steinberg, D. 1998. Evidence that the lipid moiety of oxidized low density lipoprotein plays a role in its interaction with macrophage receptors. Proc. Natl. Acad. Sci. USA. 95:1806-1811.
38. Marinetti, G. 1962. Chromatographic separation, identification, and analysis of phosphatides. J. Lipid Res. 3:1-20.

39. Reaven, P., et al. 1993. Effects of oleate-rich and linoleate-rich diets on the susceptibility of low density lipoprotein to oxidative modification in mildly hypercholesterolemic subjects. J. Clin. Invest. 91:668-676.

40. Quehenberger, O., Jurgens, G., Zadravec, S., and Esterbauer, H. 1988. Oxidation of human low density lipoprotein initiated by copper(II)chloride. Basic Life Sci. 49:387-390.

41. Chomczynski, P., and Sacchi, N. 1987. Single-step method of RNA isolation by acid guanidinium thiocyanate-phenol-chloroform extraction. Anal. Biochem. 162:156-159.

42. Tangirala, R.K., Murao, K., and Quehenberger, O. 1997. Regulation of expression of the human monocyte chemotactic protein-1 receptor (hCCR2) by cytokines. J. Biol. Chem. 272:8050-8056.

43. Watson, A.D., et al. 1997. Structural identification by mass spectrometry of oxidized phospholipids in minimally oxidized low density lipoprotein that induce monocyte/endothelial interactions and evidence for their presence in vivo. J. Biol. Chem. 272:13597-13607.

44. Hörkkö, S., et al. 1999. Monoclonal autoantibodies specific for oxidized phospholipids or oxidized phospholipid-protein adducts inhibit macrophage uptake of oxidized low-density lipoproteins. J. Clin. Invest. 103:117-128.

45. Boullier, A., et al. 2000. The binding of oxidized low density lipoprotein to mouse CD36 is mediated in part by oxidized phospholipids that are associated with both the lipid and protein moieties of the lipoprotein. J. Biol. Chem. 275:9163-9169.

46. Nagy, L., Tontonoz, P., Alvarez, J.G., Chen, H., and Evans, R.M. 1998. Oxidized LDL regulates macrophage gene expression through ligand activation of PPARgamma. Cell. 93:229-240.

47. Huang, J.T., et al. 1999. Interleukin-4-dependent production of PPARgamma ligands in macrophages by 12/15-lipoxygenase. Nature. 400:378-382.

48. Nolte, R.T., et al. 1998. Ligand binding and co-activator assembly of the peroxisome proliferator-activated receptor-gamma. Nature. 395:137-143.

49. Yamada, Y., Doi, T., Hamakubo, T., and Kodama, T. 1998. Scavenger receptor family proteins: roles for atherosclerosis, host defense and disorders of the central nervous system. Cell. Mol. Life Sci. 54:628-640.

50. Forman, B.M., Chen, J., and Evans, R.M. 1997. Hypolipidemic drugs, polyunsaturated fatty acids, and eicosanoids are ligands for peroxisome proliferator-activated receptors alpha and delta. Proc. Natl. Acad. Sci. USA. 94:4312-4317.

51. Benz, D.J., et al. 1995. Enhanced levels of lipoperoxides in low density lipoprotein incubated with murine fibroblast expressing high levels of human 15-lipoxygenase. J. Biol. Chem. 270:5191-5197.

52. Folcik, V.A., Nivar-Aristy, R.A., Krajewski, L.P., and Cathcart, M.K. 1995. Lipoxygenase contributes to the oxidation of lipids in human atherosclerotic plaques. J. Clin. Invest. 96:504-510.

53. Yamashita, H., Nakamura, A., Noguchi, N., Niki, E., and Keuhn, H. 1999. Oxidation of low density lipoprotein and plasma by 15 -lipoxygenase and free radicals. FEBS Lett. 445:287-290.

54. Zhu, L., Bisgaier, C.L., Aviram, M., and Newton, R.S. 1999. 9-cis retinoic acid induces monocyte chemoattractant protein-1 secretion in human monocytic THP-1 cells. Arterioscler. Thromb. Vasc. Biol. 19:2105-2111.

55. Nozaki, S., et al. 1995. Reduced uptake of oxidized low density lipoproteins in monocyte-derived macrophages from CD36-deficient subjects. J. Clin. Invest. 96:1859-1865.

56. Febbraio, M., et al. 1999. A null mutation in murine CD36 reveals an important role in fatty acid and lipoprotein metabolism. J. Biol. Chem. 274:19055-19062.

57. Greenwalt, D.E., et al. 1992. Membrane glycoprotein CD36: a review of its roles in adherence, signal transduction, and transfusion medicine. Blood. 80:1105-1115. 